\title{
Paciente adolescente masculino con clínica de fiebre, pérdida de peso y epigastralgia durante 6 meses
}

\author{
Jean Pierre Lucas Alarcón[1], Byron Xavier Padilla Cuenca[1] \\ 1. Hospital General Santo Domingo, Médico Cirujano, Santo Domingo de los Tsáchilas - Ecuador
}

Doi: https://doi.org/10.23936/pfr.v6i2.202

PRÁCTICA FAMILIAR RURAL | Vol.6 | No.2 | Julio 2021 | Recibido: 22/05/2021 | Aprobado: 29/07/2021

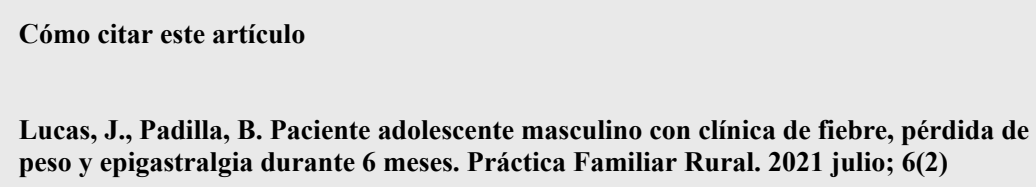

Compartir en:

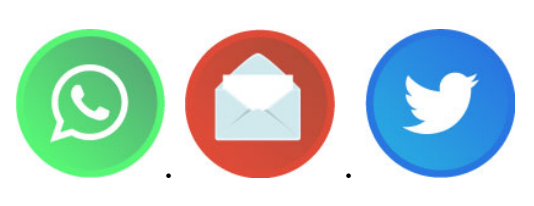

\section{Resumen}

Se presenta el caso de un paciente masculino de 19 años que acude a consulta en compañía de su madre, refiere cuadro clínico de 6 meses de evolución caracterizado por pérdida de peso, astenia y anorexia, alza térmica intermitente, epigastralgia, sensación de plenitud gástrica precoz, vómito y disfagia a los líquidos.

Palabras clave: enfermedades indiferenciadas del tejido conectivo, lupus eritematoso sistémico, nefritis lúpica, vértigo posicional paroxístico benigno.

Male adolescent patient with symptoms of fever, weight loss and epigastric pain for 6 months

\begin{abstract}
We present the case of a 19-year-old male patient who comes to the consultation in the company of his mother, reports a 6-month clinical picture characterized by weight loss, asthenia and anorexia, intermittent thermal rise, epigastric pain, a sensation of early gastric fullness., vomiting and fluid dysphagia.
\end{abstract}

Key words: undifferentiated connective tissue diseases, systemic lupus erythematosus, lupus nephritis, benign paroxysmal positional vertigo. 


\section{Presentación del caso}

Se presenta un paciente masculino de edad 19 años, nacionalidad ecuatoriana, raza mestiza, procedente y residente de Babahoyo, estado civil soltero, ocupación comerciante, instrucción educativa secundaria completa, lateralidad diestra, desconoce tipificación sanguínea.

No refiere antecedentes patológicos personales, ni quirúrgicos, ni alérgicos.

Antecedentes patológicos familiares: Madre y padre con diagnóstico de hipertensión arterial (HTA), abuelo paterno diagnosticado con cáncer pulmonar.

Hábitos tóxicos: Refiere consumir marihuana una vez al día por los últimos 6 meses.

Acude a consulta en compañía de su madre, el cual refiere cuadro clínico de 6 meses de evolución aproximadamente, caracterizado por pérdida de peso de más $15 \mathrm{~kg}$ sin causa aparente acompañado de astenia y anorexia; hace un mes presenta alza térmica no cuantificada de forma intermitente que no cede a la administración de antipiréticos además refiere epigastralgia, sensación de plenitud gástrica precoz, náuseas que en algunas ocasiones llegan al vómito y los últimos días presenta disfagia a los líquidos.

Al examen físico se encuentra consciente y orientado en tiempo, espacio y persona, independiente de necesidades básicas e instrumentales, función cognitiva conservada; llama la atención a la auscultación cardiaca soplo en foco pulmonar, con desdoblamiento de segundo ruido, mantenido a la inspiración; abdomen suave, blando, depresible, doloroso a la palpación profunda en epigastrio, no visceromegalia; región genito-inguinal se palpan adenomegalias inguinales bilaterales en número de dos, de $2 \mathrm{~cm}$ aproximadamente cada una, móvil, de bordes regulares.

Signos vitales: frecuencia cardiaca 83 lpm, frecuencia respiratoria $20 \mathrm{rpm}$, presión arterial 140/100 mmHg, saturación de oxigeno $98 \%$, temperatura 36.8 grados Celsius.

Ingresa con diagnóstico de neoplasia gástrica a estudiar (C169), fiebre no especificada (R509) e hipertensión arterial (I10). Se solicitan complementarios.

- Electrocardiograma que no reporta alteraciones: Frecuencia cardiaca 68 lpm, ritmo sinusal, eje -30 .

- Radiografía de tórax con derrame pleural bilateral de escaso contenido.

- Biometría hemática: Glóbulos blancos 3.09 K/uL (4 - 10), hemoglobina 10.2 g/dL (13 - 17), hematocrito $28.2 \%(40-54)$, volumen corpuscular medio $87.8 \mathrm{fl}(80-100)$, hemoglobina corpuscular media 31.8 pg $(27$ - 34), plaquetas $264 \mathrm{~K} / \mathrm{uL}(150-450)$, compatible con leucopenia y anemia normocitica normocrómica leve.

- Bioquímica sanguínea: glucosa basal $70.4 \mathrm{mg} / \mathrm{dL}(70$ - 106), urea $15.5 \mathrm{mg} / \mathrm{dL}(15$ - 50), creatinina $1.02 \mathrm{mg} / \mathrm{dL}(0.80-1.30)$, fosfatasa alcalina $237 \mathrm{U} / \mathrm{L}(53-270)$, aspartato aminotransferasa (AST) $67.1 \mathrm{U} / \mathrm{L}(0-40)$, alanina aminotransferasa (ALT) $52.8 \mathrm{U} / \mathrm{L}(0-45)$, proteína total en suero $4.27 \mathrm{~g} / \mathrm{Dl}$ $(6.40-8.30)$, albumina en suero $2.23 \mathrm{~g} / \mathrm{dL}(3.50-5.50)$.

- Serología: VDRL no reactivo, HIV no reactivo.

- Urianálisis: Proteinuria (+) $30 \mathrm{mg} / \mathrm{dL}$, sangre (++) 50 Ery/uL, microscopia con hematíes 48-50/campo; proteinuria en orina en 24 horas $3556 \mathrm{mg} / 24(<100 \mathrm{mg} /$ día $)$.

- Ecografía abdominal que evidencia mucosa gástrica con un grosor de $0.60 \mathrm{~cm}$, sugestivo de gastritis.

- Tomografía abdominopélvica que posterior a la administración del medio de contraste no se evidencia cambios patológicos.

- No se puede realizar videoendoscopia digestiva alta (VEDA) por no disponer con el servicio en nuestro nivel de atención, se solicita estudio por medio de trabajo social a un nivel de mayor complejidad, con respuesta desfavorable.

- Marcadores oncológicos; alfa feto proteína en $1.81 \mathrm{ng} / \mathrm{dL}(<7)$, antígeno carcinoembrionario $(\mathrm{CEA})$ $0.58 \mathrm{ng} / \mathrm{mL}(0.0-3.8)$.

Por la presentación clínica de HTA, hematuria y proteinuria se lo encasilla como un síndrome nefrítico, por lo cual se le administra antihipertensivo tipo inhibidor de la enzima convertidora de angiotensina (IECA). 
Por la manifestación de fiebre y pérdida de peso en un período de 6 meses, se propuso como una posible causa etiológica una infección primaria por tuberculosis, entidad presente en algunas regiones del país, sin embargo el paciente no manifiesta sintomatología respiratoria ni otros síntomas generales de esta patología de consideración dentro de los criterios clínicos; con respecto a los criterios de imagen, la radiografía si muestra un derrame pleural bilateral de escaso contenido, pero no hay compromiso de la trama bronquial o el parénquima pulmonar; en los criterios epidemiológicos no es una persona vulnerable y no presenta factores de riesgo asociados a contraer la enfermedad; por lo cual de acuerdo con los lineamientos establecido por el Ministerio de Salud Pública del Ecuador (MSP) en la "Guía para la Prevención, Diagnóstico, Tratamiento y Control de Tuberculosis" se descarta tuberculosis pulmonar por lo cual no amerita realización de pruebas enfocadas en esta patología.

En su estancia hospitalaria se agrega a clínica deposiciones en escala de Bistrol tipo VI en número 5 a 8 veces al día, sin presencia de hematemesis, melena o hematoquecia; manifiesta además vértigo relacionados con el cambio de posición, episodios se intensifican al momento de levantarse de la cama, maniobra de Pagnini-McClure observando nistagmo horizontal de predominio en ojo derecho, catalogándolo como vértigo postural paroxístico benigno del canal semicircular horizontal (VPPB-CH).

Al 5 día de su hospitalización aparece eritema que afecta región de mejillas y puente de la nariz dispuesto en alas de mariposa (Imagen I), se solicita biometría hemática de control y anticuerpos antinucleares.

- Biometría hemática: glóbulos blancos $3.96 \mathrm{~K} / \mathrm{uL}(4-10)$, hemoglobina $11 \mathrm{~g} / \mathrm{dL}$ (13 - 17), hematocrito $31.7 \%(40-56)$, volumen corpuscular medio $78.9 \mathrm{fl}(80-100)$, hemoglobina corpuscular media 28.1 pg $(27$ - 34), plaquetas $225 \mathrm{~K} / \mathrm{uL}(150$ - 450), compatible con leucopenia y anemia microcítico normocrómica leve.

- Anticuerpos antinucleares (ANA) 10.4 ratio $(<1.0)$.

\section{Imagen 1.- Eritema malar dispuesto en alas de mariposa}

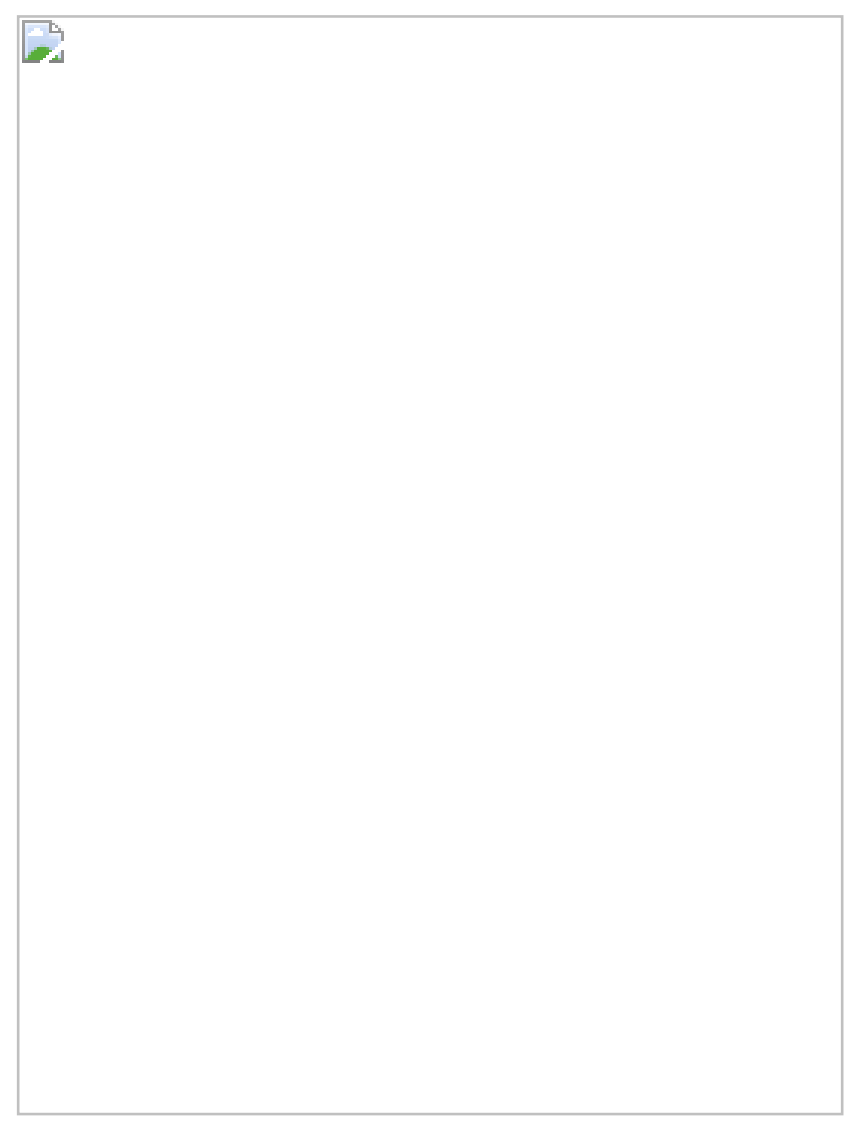

Fuente: los autores

Se descarta neoplasia gástrica, con un ANA positivo instaura el diagnóstico presuntivo de conectivopatía, se solicita exámenes inmunitarios. 
- Coombs directo: Positivo, compatible con hemolisis autoinmune.

- Anti-Smith $>200 \mathrm{U} / \mathrm{mL}(<15)$

- Anti-MPO (pANCA) $1.20 \mathrm{U} / \mathrm{mL}(<5)$.

- Anti-PR3 (cANCA) $1.40 \mathrm{U} / \mathrm{mL}(5)$.

- Anticoagulante lúpico LA1 $94.2 \mathrm{seg}$. (30 - 45), anticoagulante lúpica LA2 $39.3 \mathrm{seg}$. (31 - 39), razón LAC $1.51(<1.2)$, anticoagulante lúpico LA1 Mix 74.6 seg. $(30-40)$, anticoagulante lúpico LA2 Mix 36.4 seg. $(31-39)$.

Se establece el diagnóstico definitivo de conectivopatía tipo lupus eritematosa sistémica (LES), en concordancia a los criterios de la Liga Europea contra el Reumatismo (EULAR)/Colegio Americano de Reumatología (ACR) para LES 2019 (Anexo I) contando con un puntaje total de 29 (fiebre 2 pts., hemolisis autoinmune 4 pts., lupus cutáneo agudo 6 pts., derrame pleural 5 pts., proteinuria $>0.5 \mathrm{~g} /$ día 4 pts., anticoagulante lúpico 2 pts., anticuerpo anti-Sm 6 pts.), se relaciona el daño renal como una afección visceral por LES, instaurando también el diagnóstico de Nefritis Lúpica de acuerdo a los criterios ya expuestos. Se inicia tratamiento con metilprednisolona $1 \mathrm{~g} \mathrm{IV}$ una vez al día por tres días, posteriormente se indica ciclofosfamida $1000 \mathrm{mg}+$ dextrosa $5 \%$ en cloruro de sodio $0.9 \% 300 \mathrm{ml}$ pasar IV en 3 horas lento dosis única, después de tratamiento se realiza complementarios de control.

- Biometría hemática: glóbulos blancos $7.02 \mathrm{~K} / \mathrm{uL}(4-10)$, hemoglobina $15.9 \mathrm{~g} / \mathrm{dL}$ (13 - 17), hematocrito $46.1 \%(40-56)$, volumen corpuscular medio $87.9 \mathrm{fl}(80-100)$, hemoglobina corpuscular media 30.3 pg $(27$ - 34), plaquetas $328 \mathrm{~K} / \mathrm{uL}(150-450)$.

- Bioquímica sanguínea: urea $32.8 \mathrm{mg} / \mathrm{dL}(15-50)$, creatinina $0.67 \mathrm{mg} / \mathrm{dL}(0.80-1.30)$, AST $17.2 \mathrm{U} / \mathrm{L}$ $(0-40)$, ALT 13.3 U/L $(0-45)$.

- Proteinuria en orina en 24 horas reporta $3223 \mathrm{mg} / 24$ horas.

El paciente presenta adecuada respuesta a tratamiento y evolución favorable de clínica, se decide alta médica con tratamiento ambulatorio y controles, se recomienda realizar biopsia renal.

\section{Diagnósticos definitivos:}

- Lupus eritematoso sistémico

- Nefritis lúpica.

\section{Discusión}

Las enfermedades indiferenciadas del tejido conectivo son un grupo heterogéneo de enfermedades emparentadas que comparten características clínicas y patológicas similares de inflamación generalizada que afectan de forma difusa al tejido conectivo ocasionadas por fenómenos de autoinmunidad, desarrollando autoanticuerpos o células $\mathrm{T}$ autorreactivas afectando diferentes órganos y sistemas, se desconoce todavía el origen de este fenómeno, por este motivo los signos y síntomas que se presentan suelen ser muy inespecíficos pero en muchos casos presentan síndrome constitucional, fiebre o linfadenopatías.(1)

El LES se encuentra encasillada dentro de las conectivopatías autoinmunitarias no organoespecífica, el cual daña diversos órganos mediante autoanticuerpos y complejos inmunitarios. En la mayoría de los pacientes, hay autoanticuerpos años antes de que aparezca el primer signo clínico.(2)

Esta entidad tiene una prevalencia alta en mujeres y en población de raza negra, a nivel mundial se estima que la prevalencia de LES se encuentra entre el 30 y 150 por cada 100.000 habitantes, con una incidencia que varía entre $2 \cdot 2$ y $23 \cdot 1$ por 100.000 habitantes/año.(3) Por la infrecuencia de LES en la población masculina no suele ser un diagnóstico presuntivo habitual.

El síndrome constitucional está básicamente definido por la presencia de la tríada sintomática de astenia, anorexia y adelgazamiento (pérdida de peso involuntaria) es preciso una disminución de al menos el 5\% del peso corporal en al menos 3 meses. Aproximadamente el 25\% de las pérdidas ponderales involuntarias son atribuidas a una neoplasia, siendo en su mayoría de origen gastrointestinal.(4)

Así, el presente caso clínico al debutar con síndrome constitucional, signos y síntomas digestivos, se instaura al momento de su ingreso el diagnóstico presuntivo de neoplasia gástrica, encaminando los estudios 
complementarios para la valoración del mismo, siendo descartado tanto por exámenes oncológicos e imagenológicos y la clínica inusual que presenta el paciente en la estancia hospitalaria, con alta sospecha de una conectivopatía se solicita la realización de un ANA, mismo que se reporta positivo, por lo cual los siguientes complementarios se encaminan al estudio de LES, el diagnóstico de esta entidad representa un verdadero reto para el médico ya que se toma en consideración la epidemiología, síntomas y signos que suelen ser muy variados e inespecíficos y excluyendo diagnósticos alternativos, por lo cual los nuevos criterios EULAR/ACR para LES son una herramienta muy útil en la actualidad.

Los criterios de clasificación establecidos por el Colegio Americano de Reumatología (ACR) y por el grupo Clínicas de Colaboración Internacional contra el Lupus Sistémico (SLICC) han quedado ya relegados por los criterios de la EULAR/ACR, estos nuevos criterios parten de la presencia de ANA $>1: 80$ en células HEp-2 o una prueba equivalente positiva y tomando en consideración 7 dominios clínicos y 3 inmunológicos, cada dominio tiene criterios cuyo puntaje oscila entre 2 y 10; el SCORE clasificara como conectivopatía tipo LES a todo paciente que tengan igual o más de 10 puntos y cumpla un criterio clínico. Estos nuevos criterios presentan una sensibilidad 96,1\% y una especificidad de 93,4\%.(5)

Es infrecuente pero no improbable que se presente LES en la población masculina ya que este es más frecuente en mujeres con una proporción de 9:1 frente a varones, sobre todo en mujeres en edad fértil,(3) además es inusual que esta entidad se manifieste principalmente con signos y síntomas digestivos inespecíficos ya que dicha manifestación clínica tienen solo una prevalencia del $30 \%$ en los pacientes diagnosticados con LES.(2)

El sistema vestibular parece verse afectado aunque es atípica la presencia de VPPB en pacientes diagnosticados con LES, al momento no se cuenta con suficiente bibliografía que explique la relación. Un estudio reciente sobre "Riesgo de VPPB en pacientes con trastornos depresivos" dio como resultado que LES se identificó como un factor de riesgo independiente para desarrollar VPPB en esta población, se ha observado que la desregulación del sistema inmunológico es una parte esencial de la patogénesis del VPPB, sugiriendo que se podría desarrollar por un ataque directo o indirecto del sistema inmunológico.(6) Esta explicación podría darnos pautas para entender la manifestación de VPPB en LES y algunas otras enfermedades autoinmunitarias de presentación agresiva, ya que la desregulación del sistema inmune daría como resultado autoanticuerpos que atacarían los antígenos presentes en el oído interno.

En LES el daño renal es altamente problemático, siendo así la nefritis lúpica la enfermedad visceral más común. Un urianálisis alterado con o sin una concentración de creatinina plasmática elevada está presente en una gran proporción de pacientes, el hallazgo que se observa con mayor frecuencia en el urianálisis de pacientes con LES es la proteinuria, de hecho, si se hace biopsia renal en todos los afectados con LES, aproximadamente el $90 \%$ presentan algún tipo de lesión renal,(7) por eso en los nuevos criterios de EULAR/ACR para LES se considera diagnóstico de nefritis lúpica todo paciente con ANA positivo y la aparición de proteinuria $>0,5 \mathrm{~g} / 24 \mathrm{~h},(5)$ por años el gold standard para diagnosticar la nefritis lúpica fue la biopsia renal, sin embargo se recomienda todavía la realización de la misma en todos los pacientes con nefritis lúpica para poder categorizar el grado de daño renal de acuerdo a los parámetros de clasificación actualmente vigentes de la Sociedad Internacional de Nefrología (INS) y Sociedad de Patología Renal (RPS).

En el tratamiento para LES los corticosteroides ejercen una potente acción antiinflamatoria y efectos inmunosupresores, pese esto el consumo prolongado y crónico ejercen muchos efectos secundarios,(3) con respecto a los signos y síntomas digestivos y el aumento de las transaminasas (ALT y AST) mejoran luego de la administración de corticosteroides,(2) además en el tratamiento de los esquemas clásico para la nefritis lúpica se basaban en la administración de ciclofosfamida y esteroides, aunque estos tratamientos han demostrado un menor riesgo de progresión hacia la insuficiencia renal, tienen varios efectos secundarios de considerable importancia.(7)

\section{Conclusiones}

- Se analiza LES en un paciente masculino en la etapa de la adolescencia de raza no negra, con signos y síntomas predominantemente digestivos, la inusual y poco documentada manifestación de VPPB en esta entidad, por lo cual se lo considera una presentación atípica de LES. 
- Considerar el uso de los criterios propuestos por EULAR/ACR como una nueva herramienta que facilita identificar LES.

- La biopsia renal deja de ser el gold standard para diagnosticar nefritis lúpica en concordancia con los criterios EULAR/ACR, mas se recomienda la realización de la misma para determinar la magnitud del daño renal.

- La importancia de realizar un ANA en pacientes con alta sospecha de conectivopatía, además de ser el pilar del cual parten los nuevos criterios EULAR/ACR.

- Parece existir una relación entre LES y la presencia de VPPB aunque su fisiopatología aún no es clara y se necesitan más estudios.

\section{Referencias bibliográficas}

- Clemente D, López J. Conectivopatías en Adolescentes. Adolescere. 2015; III (1): 30 - 43.

- Hannahs B. Lupus Eritematoso Sistémico. En: Kasper D, Longo D, Fauci A, Hauser S, Jameson J, Loscalzo J, editores. Harrison - Principios de Medicina Interna. Vol 2. $19^{\circ}$ ed. México: McGraw-Hill; 2015. p. $2124-2134$.

- Durcan L, O'Dwyer T, Petri M. Management Strategies and Future Directions for Systemic Lupus Erythematosus in Adults. Lancet. 2019; 393 (10188): 2332 - 2343.

- Suárez S, Puente A, Santana S, Díaz G, Fuente S, Peláez S. Síndrome Constitucional: Entidad Clínica o Cajón de Sastre. Rev Med Inst Mex Seguro Soc. 2013; 51 (5): 532 - 535.

- Wallace D, Gladman D. Clinical Manifestations and Diagnosis of Systemic Lupus Erythematosus in Adults. Up-to-date [Internet]. 2019. [citado 05 Mar 2021]. Disponible en: https://www.uptodate.com

- Hsu CL, Tsai SJ, Shen CC, Lu T, Hung YM, Hu LY. Risk of Benign Paroxysmal Positional Vertigo in Patients with Depressive Disorders: a Nationwide Population-Based Cohort Study. BMJ Open. 2019; $9(3): 1-9$

- Rivera F, Romera A, Villabón P, Sánchez P, Anaya S, González L, et al. Lupus Eritematoso Sistémico - Nefropatía Lúpica. Nefrología al Día [Internet]. 2020. [citado 05 Mar 2021]. Disponible en: https://www.nefrologiaaldia.org 